\title{
Effects of daily timing of saturated fat and liquid sugar intake on energy balance
}

\author{
J.E. Oosterman ${ }^{1}$, L.L. Koekkoek ${ }^{1}$, E. Foppen ${ }^{1}$, E. Fliers ${ }^{1}$, S.E. la Fleur ${ }^{1}$ and A. Kalsbeek ${ }^{1,2}$ \\ ${ }^{1}$ Department of Endocrinology and Metabolism, Academic Medical Center, University of Amsterdam, Amsterdam, \\ The Netherlands and ${ }^{2}$ Hypothalamic Integration Mechanisms, The Netherlands Institute for Neuroscience, Amsterdam, \\ The Netherlands
}

Food intake during the inactive or sleep period can lead to uncoupling of peripheral clocks from the central clock in the suprachiasmatic nucleus ${ }^{(1)}$, and to desynchronization between clock genes and clock-controlled genes within one organ, possibly contributing to metabolic disorders ${ }^{(2)}$. Meal composition can also disrupt circadian rhythms ${ }^{(3)}$, but at present, it is unknown if the inappropriate timing of specific diet components (e.g. fat or sugar) is responsible for alterations in body weight or metabolism.

In the present study, we therefore studied (i) the effect of eating palatable food at an 'inappropriate' time of day, i.e. the normally inactive period, on energy balance and metabolism, and (ii) the effect of these diets on the rhythmic expression of clock genes and clock-controlled genes in the liver. Male Wistar rats $(\mathrm{n}=258)$ were subjected to a chow, a high-fat high-sugar $(\mathrm{HFHS})$, or a high-fat (HF) diet, either ad libitum, or with restricted access to the diet for 10 hours during light (L) or dark (D) phase. Food intake and body weight were monitored during five weeks. Energy expenditure was measured in metabolic cages in the fourth week. Afterwards, serum was collected and tissues were dissected.

Caloric food intake was significantly higher in all groups receiving fat and/or sugar compared to the chow groups $(\mathrm{P}<0 \cdot 05)$. Caloric food intake in the HFHS L group was lower than in the HFHS ad lib group $(\mathrm{P}<0.05)$. Timing did not affect body weight gain or adiposity within diet groups, but it affected hepatic fat accumulation. Hepatic fat accumulation in the chow L group was significantly lower than in the chow ad lib group $(\mathrm{P}<0.01)$, whereas it was higher in the HFHS L group than in the HFHS ad lib group (P<0.05). Respiratory exchange ratio (RER) patterns in all L-groups were shifted by 12 hours, and showed greater amplitudes compared to the ad lib groups, suggesting a shift in daily substrate oxidation (Fig. 1). The HFHS ad libitum diet led to an amplitude dampening in the circadian expression of Bmall and Per2 in the liver, whereas the restricted diets $-\mathrm{L}$ or $\mathrm{D}$ - strengthened the amplitude. Furthermore, the restricted diets led to a rhythm shift compared to the ad libitum groups.

We conclude that restricting food to either the light or dark period, leads to alterations in hepatic fat accumulation, perhaps due to a shift in substrate oxidation. Furthermore, food restriction has a strong effect on rhythmic clock gene expression in the liver. Further analysis is underway to assess the effects of differently timed diets on clock-controlled genes in the liver, and the effects on alterations in oxidation patterns.

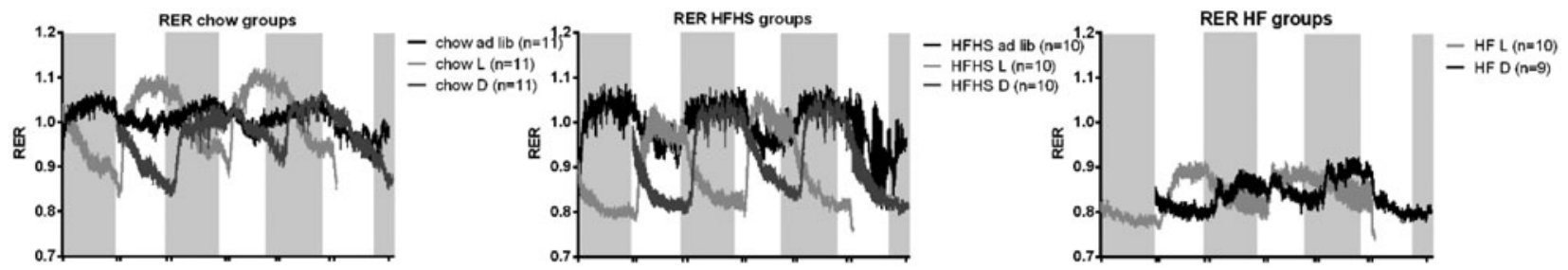

Fig 1. Respiratory exchange ratios (RER) patterns for all groups. Grey shadings indicates dark phase.

1. Stokkan KA, Yamazaki S, Tei H, Sakaki Y, Menaker M (2001). Science 291, 490-493.

2. Salgado-Delgado RC, Saderi N, Basualdo Mdel C, et al. (2013). PLoS One 8, e60052.

3. Kohsaka A, Laposky AD, Ramsey KM, et al. (2007). Cell Metab 6, 414-21. 